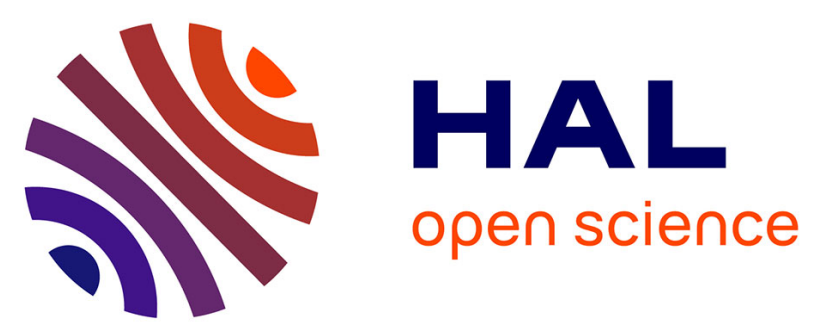

\title{
THE EFFECTS OF GRAVITATIONAL AND HYDROSTATIC PRESSURE ON THE EQUILIBRIUM SHAPE OF A CONDUCTING FLUID IN AN ELECTRIC FIELD : APPLICATION TO LIQUID METAL ION SOURCES
}

P. Cutler, M. Chung, T. Feuchtwang, E. Kazes

\section{To cite this version:}

P. Cutler, M. Chung, T. Feuchtwang, E. Kazes. THE EFFECTS OF GRAVITATIONAL AND HYDROSTATIC PRESSURE ON THE EQUILIBRIUM SHAPE OF A CONDUCTING FLUID IN AN ELECTRIC FIELD: APPLICATION TO LIQUID METAL ION SOURCES. Journal de Physique

Colloques, 1986, 47 (C2), pp.C2-87-C2-93. 10.1051/jphyscol:1986213 . jpa-00225644

\section{HAL Id: jpa-00225644 https://hal.science/jpa-00225644}

Submitted on 1 Jan 1986

HAL is a multi-disciplinary open access archive for the deposit and dissemination of scientific research documents, whether they are published or not. The documents may come from teaching and research institutions in France or abroad, or from public or private research centers.
L'archive ouverte pluridisciplinaire HAL, est destinée au dépôt et à la diffusion de documents scientifiques de niveau recherche, publiés ou non, émanant des établissements d'enseignement et de recherche français ou étrangers, des laboratoires publics ou privés. 
THE EFFECTS OF GRAVITATIONAL AND HYDROSTATIC PRESSURE ON THE EQUILIBRIUM SHAPE OF A CONDUCTING FLUID IN AN ELECTRIC FIELD : APPLICATION TO LIQUID METAL ION SOURCES

\author{
P.H. CUTLER, M. CHUNG ${ }^{+}$, T.E. FEUCHTWANG ${ }^{+}$and E. KAZES ${ }^{+}$ \\ University of Oxford, Department of Metallurgy and Science of \\ Materials, Parks Road, GB-Oxford oxi 3PH, Great Britain \\ + Department of Physics, The Pennsylvania State University. \\ University Park, Pennsylvania 16802, U.S.A.
}

\begin{abstract}
We have derived a partial differential equation that can be solved explicitly for the equilibrium shape of an electrostatically stressed fluid subject to gravitational and hydrostatic pressure effects. The model assumes only axial symmetxy, the Laplace stress conditions for mechanical equilibrium and conservation of volume before onset of instability. To obtain the sequence of deformed surfaces as a function of applied voltage, we use the capillary wave model and an iterative procedure to solve Laplace's equation for arbitrary geometry. Initial numerical results demonstrate the importance of pressure in obtaining stable equilibrium configurations.
\end{abstract}

\title{
I - INTRODUCTION
}

A fundamental problem in the theory of operation of field emission liquid metal ion sources (LMIS) is to predict the allowed static and dynamic (equilibrium) configuration(s) of the liquid enitter subject to strong electric fields. It has generally been assumed that, based on the work of Taylor $/ 1 /$, the static equilibrium shape of the conducting fluid just prior to or at the onset of instability is a cone of specified angle. However, it has never been proven theoretically or unequivocally demonstrated experimentall ${ }^{\dagger}$ that the idealized conical shape is the only, or even

\footnotetext{
TIt is illuminating to review some of Taylor's own thoughts which seem relevant to this point. In an equally important but less quoted paper published in 1969 /2/, Taylor did the following experiment. He applied an electric field normal to a fluid in a capillary tube and determined the potential necessary for the onset of instability. The meniscus was kept in an approximately constant hemispherical shape by varying the (gravitational) pressure as the field, E, is increased. The force equation for equiliorium of the meniscus is

$$
\text { Furface tension }+F_{\text {gravity }}-F_{\text {electrical }}-F_{\text {hydrostatic pressure }}=0 \text {. }
$$

More explicity
} 
rigorously correct, allowed geometry for an electrostatically stressed conducting fluid. Nevertheless much experimental /3/ and theoretical /4-6/ progress has been made on elucidating the shape of the emitting region of an LMIS. In particular the TEM images by Sudraud and co-workers /7/ have provided the first reliable evidence of the profiles of LMIS at low emission currents. Theoretical models have been developed using the ideal Taylor conical geometry /6/, or some simplified variant of it /4-6,8/ to explain certain static and dynamic features of the device. Almost always, however, pressure effects have not been included in these models.

However, there are other operating characteristics of an LMIS, whose specifications require a more accurate description of the geometric features of the emitting region than current theories can now predict. Probably the most important physical parameter which requires quantitative accuracy is the electric field at the apex of the device. This is, for example, crucial to

i) a correct theory of the ion formation mechanism $/ 4,8 /$

ii) virtual source size calculations $/ 9,10 \%$

In addition, the electrohydrodynamics of conducting droplets and fluids has many diverse applications $/ 11,12 /$, of which the field emission LMIs is only one. For many, of these applications, a rigorous and accurate treatment of equilibrium and dynamic properties, including the shape of the stressed fluid body, is important. In this paper we have derived a partial differential equation that can be solved explicitly for the equilibrium shape of an electrostatically stressed fluid subject to gravitational and hydrostatic pressure effects. To numerically solve for the sequence of deformed surfaces as a function of the applied electric field, we have used the results of the capillary wave model as an initial configuration and an iterative procedure due to Melcher /13/ to solve Laplace's equation for an arbitrary shape of the fluid interface. The preliminary numerical results demonstrate the importance of incluaing pressure to obtain stable equlilibrium configurations before the onset of instabilities.

In Section II we review briefly the treatment of pressure in previous models. The partial differential equation is derived in Section III. Our initial results and conclusions are given in Section IV.

\section{II - TREATMENT OF PRESSURE IN PREVIOUS THEORIES}

In this section we shall review the treatment of pressure in the Tayior theory /1,2/ and the variational formulation for the equilibrium shape of a stressed conducting fluid developed by the authors $/ 14 /$.

$$
F_{\text {surface tension }}+W=\frac{E^{2}}{8 \pi}+\pi R^{2} p_{H}
$$

where $w$ is the weight of the fluid in the droplet, $R$ the radius of the meniscus and $p_{H}$ the hydrostatic pressure. Taylor concluded "That in all cases where the meniscus was initially hemispherical, the value of $p_{H}$ at breakdown is small compared with its initial value..." Although he never provides any numerical estimates, it is possible to show from the published data that at breakdown

$$
\mathrm{P}_{\mathrm{H}}(\text { breakdown }) \approx 10^{-1} \mathrm{P}_{\mathrm{H}} \text { (initial) }
$$

Taylor further asserts, "...that when $p=0$ (i.e., near or at onset of instability) the meniscus is nearly conical with a semi-vertical angle close to the only possible equilibrium value of $49.3^{\circ}$ (Taylor, 1964) and it will be noticed that the surface remains nearly conical at the outer edge of the tube, though this cannot be an accurate result derivable from theory." (Underlining is ours.)

The significance of this last statement is that to obtain a true (Taylor) cone, you must have a straight line generator for the cone at the appropriate angle of $49.3^{\circ}$, otherwise a surface results with either convex or concave curvature. 
Based on observations, Tayior assumed an ideal right circular cone for the 'meniscus' profile of a conducting fluid in an electric field. Using essentially dimensionality arguments, Taylor derived an equilibrium condition (for notation see Ref. /1/, or $/ 14 /)$

$$
\frac{Y \cot \alpha}{R}=\frac{1}{8 \pi}\left(\frac{1}{R} \frac{d V}{d \theta}\right)_{\theta=\pi-\alpha}^{2}
$$

which is valid only for

i) A specially defined geonetry of the fluid (i.e., conical) and curved counterelectrode (i.e., $\left.R=R_{0}\left[P_{1 / 2}(\cos \theta)\right]^{-2}\right)$,

and

ii) zero hydrostatic pressure.

That is, Taylor assumed that, near or at onset or instability, the hydrostatic pressure is negligible--or even exactly zero. This latter result is never proven. An analysis of the 1969 paper, $/ 2 /$, suggests that Taylor used the simplifying assumption of a "small" pressure at breakdown to be identically zero. However, our results indicate that even small values of $\Delta \mathrm{pH}$ can be significant in determining the deformation of fluids at higher valves of the electric field. It has been suggested, in justification of the Taylor model that, "... pressure is not a quantity which one derives but is an experimental parameter. In principle, however, one can chose to consider the caser where $\mathrm{p}_{1}=\mathrm{p}_{2}\left(\mathrm{i}, \mathrm{e},, \Delta \mathrm{p}_{\mathrm{H}}=0\right)$; there is no intrinsic reason why $P_{1}$ and $p_{2}$ must differ in the case of an electrically charged surface." /15/, or "... the reason Taylor set $\Delta \mathrm{pH}_{\mathrm{H}}=0$ is simply he was looking for solutions fulfilling this condition.." /16/. With respect to the latter comment we have already indicated above the possible significance of small absolute values of $\Delta \mathrm{pH}_{\mathrm{H}}$.

In response to the former, we note that the hydrostatic pressure difference is one of the intrinsic (intermolecular) forces that determine the mechanical stability of a fluid subject to capillary, gravitational, electrical or other external force fields. For equilibrium the forces on the fluid must satisfy

$$
\sum F_{\dot{S}}^{\prime}=F_{\Delta p_{H}}+F_{\text {electrical }}-F_{\text {surface tension }}-F_{\text {gravitational }}+\ldots=0 \text {. }
$$

Obviousiy $\Delta_{\mathrm{p}_{\mathrm{H}}}$ is not an independent quantity and it cannot be arbitrarily set equal to zero or any other value. Furthermore, in experiments observing stressed fluid shapes, one can control the applied voltage, the gravitational pressure, the surface tension and other external forces; however, for a fluid surface with non-zero curvature, it is not possible, in general, to experimentally make $\Delta \mathrm{p}_{\mathrm{H}}=0$.

\section{II.B - Variational Formulation}

We have previously used the principle of energy minimization to derive an equilibrium--or stress balance--condition for a conducting fluid in an electric field $/ 14,17 /$. The resulting set of coupled Euler-Lagrange equations include a Lagrangian multiplier $\lambda$. In the past we were not able to provide a convincing physical interpretation of $\lambda$, except to say that it was identified with the gravitational and hydrostatic pressure. We have now shown explicity that $\lambda=\Delta \mathrm{p}$ where $\Delta \mathrm{p}$ is the total plessure acting on the fluid $/ 18 /$. The analysis uses minimization of the energy of an electrostatically stressed conducting fluid, with a constant but arbitrary volume, to derive the generalized stress condition

$$
\gamma\left(\frac{1}{R_{1}}+\frac{1}{R_{2}}\right)-\frac{1}{8 \pi} \mathrm{E}_{s}^{2}+\lambda=0
$$

where notation is standard. Comparison with the Laplace stress condition immediately yields the equality of $\lambda$ and $\Delta$ p. The identical result were obtained by a Russian 
group /19/ who investigated the mechanical stability of a static axi-symmetric liquid meniscus in a non-zero gravitational field. Using a variational formalism, with the constraint that the volume is constant, they find that the Iagrangian multiplier in their Euler-Lagrange equations for determining the shape of the fluid has the physical interpretation of hydrostatic pressure in the meniscus. These results should dispel any lingering doubts about the validity of using the variational formalism, or the use of the resulting set of Euler-Lagrange equations (EL) to determine the equilibrium shape of a stressed fluid $/ 14 \mathrm{c} /$.

The BL equations can, in principle, be used to determine the equilibrium shape: One could solve analytically or numerically the set of coupled equations. The former method appears unrealistic because of the sheer complexity of the mathematical equations $/ 14 /$. A numerical solution is feasible, but neither the parameter $\lambda$ (i.e., the pressure) nor the electric field for arbitrary shape is known. A second method of solution is to use some model or empirical shape and determine whether the equations are satisfied. We have used this approach with the experimental observation of a cuspidal shape at low initial emission $/ 20 \%$. It was assumed that this might be the precursor shape just prior to the onset of instability $/ 3 /$. It was not possible to establish that the cuspidal model satisfied the EL equations; it was suggested that the difficulty might be the singular point in the ideal cusp geometry.

The inability, within this formalism--or others $/ 1,2 /-$ to include explicitly the pressure prompted the derivation of a new equation which accounts for gravitational and hydrostatic pressure effects. Since these contributions can now be computed for arbitrary deformation of the fluid, the EI equations may also be more amenable to solution by the second method described above.

\section{III - DERTVATION}

Since the detailed derivation will be given elsewhere $/ 21 /$, only a brief description of it and the iterative procedure for solving Laplace's equation will be presented here.

Consider a conducting fluid of constant volume which rests on the upper surface of a cylindrical electrode with radius a (see Fig. 1). We assume, for simplicity, that the initial shape is an ellipsoid of revolution. As the

traman coumten-ticeinoor $\therefore 0$
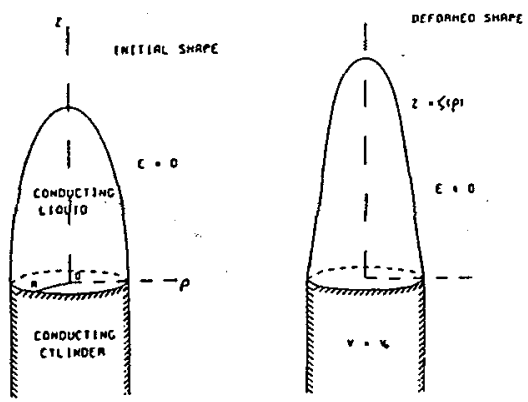

Fig. 1. Schematic depiciting fluid shape for zero field and deformation of the fluid after field is applied. Liquid and cylinder are treated as equipotential surfaces. field is increased, the surface of the fluid deforms; additional assumption of the model are

i) the equation of the surface is given by

$$
F(z, \rho)=z-\zeta(\rho)=0
$$

where $(z, \rho)$ are cylindrical coordinates.

ii) The equilibrium shape of the conducting fluid is described by the Laplace stress condition:

$$
\Delta p+2 \pi \sigma_{s}^{2}-\gamma\left(\frac{1}{R_{1}}+\frac{1}{R_{2}}\right)=0
$$

where $\mathrm{E}_{\mathrm{s}}=4 \pi \sigma_{\mathrm{s}}^{2}, \mathrm{~s}$ denotes surface and 


$$
p=\Delta p_{H}+\Delta p_{\text {gravitational }}=p_{\text {fluid }}-p_{\text {air }} \equiv p_{\text {fluid }}^{0}-\rho_{\text {fluid }} g \zeta(0)-p_{\text {air }}^{0}
$$

$\zeta(\rho)$ measures the deformation of the surface, and plluid denotes the hydrostatic pressure of the fluid different for each given shape (i.e., values of $\left.\sigma_{s}\right)$. The pressure pair outside the fluid is assumed constant for all values of the field.

iii) Their is conservation of volume so that $\int_{0}^{a} \zeta(\rho) 2 \pi \rho d \rho$ is equal to a constant, whose value depends on the choice of origin for $\zeta(\rho)$.

It can be shown $/ 22 /$ that $\left(1 / R_{1}+1 / R_{2}\right)$ can be expressed in terms of $\zeta(\rho)$ :

$$
\gamma \rho^{-1} \frac{\mathrm{d}}{\mathrm{d} \rho}\left[\rho \zeta_{\rho}\left(1+\zeta_{\rho}^{2}\right)^{-1 / 2}\right)+2 \pi \sigma_{\mathrm{s}}^{2}+\Delta \underline{\mathrm{p}}=0
$$

where $\zeta_{\rho}=\frac{d \zeta}{d \rho}$. If we multiple by $2 \pi \rho d \rho$; integrate $\int_{0}^{a}$ and use the conservation of volume condition in $i i i)$, then $\Delta p$ can be expressed in terms of other surface quantities:

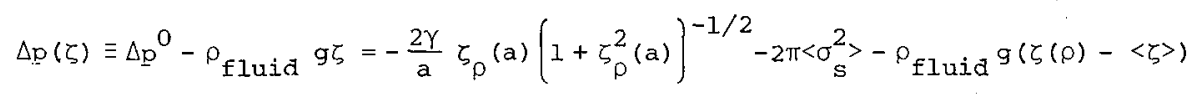

where

and

$$
\left\langle\sigma_{s}^{2}>=\frac{1}{\pi a^{2}} \int_{0}^{a} \sigma_{s}^{2} 2 \pi \rho d \rho\right.
$$

$$
\langle\zeta\rangle=\frac{1}{\pi a^{2}} \int_{0}^{a} \zeta(\rho) 2 \pi \rho d \rho
$$

Substituting into Eq. (3) yields the (non-linear) partial differential equation for $\zeta(\rho)$, the shape of the fluid:

$\gamma \rho^{-1} \frac{d}{d \rho}\left(\rho \zeta_{\rho}\left(I+\zeta_{\rho}^{2}\right)^{-1 / 2}\right)+2 \pi \sigma_{s}^{2}-\rho_{f l u i d} g \zeta=\frac{2 \gamma}{a} \zeta_{\rho}(a)\left(1+\zeta_{\rho}^{2}(a)\right)^{-1 / 2}+2 \pi\left\langle\sigma_{s}^{2}\right\rangle-\rho_{\text {fluid }} g\langle\zeta\rangle$

To solve Eq. (5) for $\zeta(\rho), \sigma_{s}$ must be known, which however depends upon the shape through Eq. (5). Therefore, an iterative procedure, due to Melcher /13/ is used to solve Laplace's equation for arbitrary geometries. The integral solution to Laplace's equation,

$$
v(\vec{r})=\int \frac{\sigma\left(\vec{r}^{\prime}\right) d A^{\prime}}{\left|\vec{r}-\vec{r}{ }^{\prime}\right|} \rightarrow v\left(\vec{r}_{m}\right) \approx \sum_{n}^{i} \frac{\Delta A_{n}}{\left|\vec{r}_{n}-\vec{r}_{m}\right|} \cdot \sigma\left(\vec{r}_{n}\right)
$$

is transformed to a sum over points on the boundaries of the fluid and the electrodes. Then, writing Eq. (6) In matrix form,

$$
v_{m}=\sum G_{m n} \sigma_{n}
$$

one solves for $\sigma_{n}=\left(G^{-1}\right\}_{m n} V_{m}$, substitutes into Eq. (5) and solves for $\zeta(P)$.

\section{IV - RESULTS AND CONCLUSIONS}

As a preliminary example of this procedure we exhibit, in Fig. 2, the numerical solutions for the evolution of a conducting fluid volume as a function of applied field. For computational convenience, the initial shape, selected at $E=0$, is an ellipsoid of revolution with semi-minor and major axes $a=20 \mu$ and $b=4 a$ respectively. This volume is about eight times that used by Taylor in his experiments in $1964 / 1 \%$, but is still small enough so that gravitational effects in the (static) equilibrium condition are negligible. This suggests that gravitational effects 
will be unimportant in the electrohydrodynamics of LMIS, where dimensions of the emitting protrusions are nearly one or more orders of magnitude smaller. By contrast,

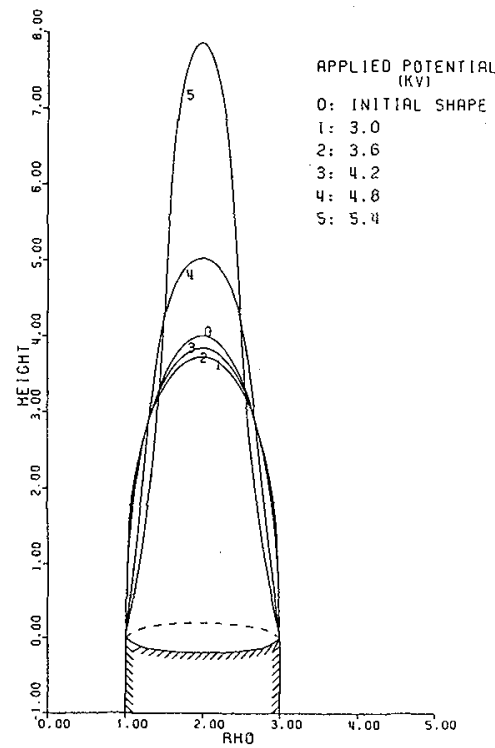

Fig. 2. Evolution of the fluid shape as a function of applied field. All profiles except that denoted by ' $O$ ' represent convergence solutions of Eq. (5). Surfaces 1 and 2 are nearly colncident. the presence of the hydrostatic pressure term is extremely, if not crucially, important in obtaining a stable solution. Whereas each of the shapes exhibited in Fig. 2 represents a stable (i.e., convergent) numerical solution of Eq. (5), usually requiring 10-15 iterations, it was not possible, in general, for a similar range of applied voltages, to obtain any stable solutions with $\Delta p_{H}$ set equal to zero. By stable solution we mean a convergent shape of the fluid meniscus, for arbitrary field and volume. However, there appears to exist for the case of constant volume, one value of shape and field, for each volume, for which the hydrostatic pressure difference ( $\Delta \mathbf{p}_{H}$ ) should be zero. This is inferred from the change of the fluid profile from convex to concave as the voltage is increased, for example, from 4.8 to $5.4 \mathrm{kV}$. This is corroborated by the computation of $\Delta \mathrm{p}_{\mathrm{H}}$ as a function of applied voltage; for low fields, $\Delta \mathrm{p}_{\mathrm{H}}$ is positive, but as the electric field stress increases and the profile elongates, the hydrostatic pressure becomes negative, consistent with the compression of the lateral dimension of the fluid shape.

We have not, in these preliminary calculations, been able to locate the precise value of field, or shape, at which $\Delta \mathrm{p}_{\mathrm{H}}$ should be zero nor do we know if this shape corresponds to an equilibrium state, or even if it is stable.

Finally, at the highest fields applied, the profiles become concave and even exhibit minima at the base corresponding to a depletion of fluid. This latter effect is an artifact of the constraint that the volume be constant. In most experimental observations of the equilibxium shape in fluids in electric fields /1,2/ or LMIS/23/, the curvature at or near the base of the fluid is convex. This suggests that fluid flow probably occurs even before onset of instability. However, we have not yet been able to assess the need of a hydrodynamic description for processes occurring at the apex region prior to or at the onset of instability (1.e., oscillations, jetting and/or neutral and charged particle emission).

In summary, we have derived a new partial differential equation, that includes the pressure explicitly, for determining the equilibrium profile of a conducting fluid in an electric field $/ 24 /$.

Preliminary results suggest the following conclusions:

i) The inclusion of $\Delta \mathrm{p}_{\mathrm{H}}$ leads to stable solutions for the shape of the fluid meniscus.

ii) It is not possible, in general, to obtain stable surfaces with $\Delta \mathrm{p}_{\mathrm{H}}=0$.

iii) For the case of constant volume a solution with $\Delta \mathrm{p}_{H}=0$ may exist but only for a special shape of the meniscus and value of the field.

iv) It is feasible to perform a rigorous numerical analysis of the evolution of the surface of a stressed conducting fluid from low fields to the onset of instabilities.

This may be interpreted as a theoretical analog of, for example, experiment TEM observations of an LMIS /3/. 
REFERENCES

*Permanent address: Department of Physics, The Pennsylvania State University, University Park, Pennsylvania 16802 U.S.A.

/1/ G. I. Taylor, Proc. Roy. Soc. Lond. 280A, 383 (1964).

/2/ Sir G. I. Taylor, Proc. Roy. Soc. Lond. A313, 453 (1969).

/3/ G. Benassayag, P. Suãraud and B. Jouffrey, Jitramicroscopy 16, 1 (1985).

/4/ D. R. Kingham and L. W. Swanson, App. Phys. A34, $123(1984)$.

/5/ R. G. Forbes, Jour. de Phys. 45, C9-161 (1984).

/6/ S. P. Thompson and P. D. Prewett, J. Phys. D: Appl. Phys. 17, 2305 (1984).

/7/ See Ref. 3 and references cited therein.

/8/ R. G. Forbes and G. L. R. Mair, J. Phys. D. Appl. Phys. 15, I153 (1982).

19/ N. M. Miskovsky, P. H. Cutler and T. E. Feuchtwang, Appl. Phys. A33, 113 (1984).

$110 /$ P. J. Hoepfner and $\mathrm{L}$. W. Swanson, Proc. of the 32 Int. Field Emission Symposium 15-19 July, 1985, wheeling, West virginia.

/11/ A list of fundamental applications, including meteorological, atmospheric, astrophysical and nuclear is given in H. H. Tang and G. Wong, J. Phys. A: Math Nucl. Gen. 7, 1038 (1984).

112/ Some recent technological applications are discussed in C. C. Mahoney. I. Gowland and P. D. Prewitt, "Liquid Metal Field Emission Systems for High Technology Applications; the FED Sprayer," Proc. of the 29th Int. Field Enission Symposium ed. by $H$. Andren and H. Norden (Almquist and Wiksell Int., Stockholm, 1982)

113/ J. B. Melcher, Continuum Electromechanics (MIT Press, Cambridge, 1981 ).

114/ a) N. Sujatha, P. H. Cutler, E. Kazes, J. P. Rogers and N. M. Miskovsky, Appl Phys. A32, 55 (1983); b) M. Chung, P. H. Cutler, T. E. Feuchtwang, N. M. Miskovsky and N. Sujatha, Scanning Electron Microscopy 1984/IV, 1547;

c) M. Chung, P. H. Cutler, T. E. Feuchtwang, E. Kazes and N. M. Miskovsky, Appl. Phys. A36, 171 (1985).

/15/ J. E. Allen, preprint and to be published J. Phys. D: Applied Physics

116/ Comment on page 1555 by Reviewer II in Ref. $14 \mathrm{~b}$.

/17/ See also, N. M Miskovsky, P. H. Cutler and E. Kazes, J. Vac. Sci. Technol. B3, 202 (1985).

/18/ Presented at the 32nd Int. Field Emission Symposium, July 15-19, Wheeling, West Virginia and to be published.

/19/ A. V. Zdhanov, G. A. Satunkin and R. P. Pononareva, Jour. Colloidal and Interface Science 104, 334 (1985).

$120 /$ M. Chung, P. H. Cutler, T. E. Feuchtwang, E. Kazes and N. M. Miskovsky, Jour. de Physique 45, C9-153 (1984).

121/ M. Chung, P. H. Cutler, T. E. Feuchtwang and E. Kazes, to be published.

/22/ Sir H. Lamb, Hydrodynamics, 6th ed., (Cambridge University Press, Cambridge (1934) p. 414 .

/23/ D. B. Kingham and A. E. Bell, Appl. Phys. A36, 67 (1985).

/24/ A. French group, using a similar variational formalism but different computational procedure, have also been calculating profiles of liquid menisci for both conducting and non-conducting fluids. Their formulation includes the gravitational but not the hydrostatic pressure contribution. Nevertheless, their method and results are very relevant to the calculations reported in this paper. See for example, G. J. Joffre, Jour. de. Mecanique theorique et appliques $3,545(1984)$ and references cited therein. 